University of Wollongong

Research Online

Faculty of Engineering and Information

Faculty of Engineering and Information

Sciences - Papers: Part A

Sciences

$1-1-2015$

\title{
Multi-view TWRI scene reconstruction using a joint Bayesian sparse approximation model
}

Van Ha Tang

University of Wollongong, vht986@uowmail.edu.au

Abdesselam Bouzerdoum

University of Wollongong, bouzer@uow.edu.au

Son Lam Phung

University of Wollongong, phung@uow.edu.au

Fok Hing Chi Tivive

University of Wollongong, tivive@uow.edu.au

Follow this and additional works at: https://ro.uow.edu.au/eispapers

Part of the Engineering Commons, and the Science and Technology Studies Commons

Research Online is the open access institutional repository for the University of Wollongong. For further information contact the UOW Library: research-pubs@uow.edu.au 


\title{
Multi-view TWRI scene reconstruction using a joint Bayesian sparse approximation model
}

\begin{abstract}
This paper addresses the problem of scene reconstruction in conjunction with wall-clutter mitigation for compressed multi-view through-the-wall radar imaging (TWRI). We consider the problem where the scene behindthe-wall is illuminated from different vantage points using a different set of frequencies at each antenna. First, a joint Bayesian sparse recovery model is employed to estimate the antenna signal coefficients simultaneously, by exploiting the sparsity and inter-signal correlations among antenna signals. Then, a subspace-projection technique is applied to suppress the signal coefficients related to the wall returns. Furthermore, a multi-task linear model is developed to relate the target coefficients to the image of the scene. The composite image is reconstructed using a joint Bayesian sparse framework, taking into account the inter-view dependencies. Experimental results are presented which demonstrate the effectiveness of the proposed approach for multi-view imaging of indoor scenes using a reduced set of measurements at each view.
\end{abstract}

\section{Keywords}

approximation, sparse, view, bayesian, multi, joint, reconstruction, scene, twri, model

Disciplines

Engineering | Science and Technology Studies

\section{Publication Details}

V. Tang, A. Bouzerdoum, S. Lam. Phung \& F. Tivive, "Multi-view TWRI scene reconstruction using a joint Bayesian sparse approximation model," in Conference on Compressive Sensing IV, 2015, pp.

948405-1-948405-10. 


\title{
Multi-view TWRI scene reconstruction using a joint Bayesian sparse approximation model
}

\author{
V. H. Tang, A. Bouzerdoum, S. L. Phung and F. H. C. Tivive \\ School of Electrical, Computer and Telecommunications Engineering, \\ University of Wollongong, NSW, 2522, Australia
}

\begin{abstract}
This paper addresses the problem of scene reconstruction in conjunction with wall-clutter mitigation for compressed multi-view through-the-wall radar imaging (TWRI). We consider the problem where the scene behindthe-wall is illuminated from different vantage points using a different set of frequencies at each antenna. First, a joint Bayesian sparse recovery model is employed to estimate the antenna signal coefficients simultaneously, by exploiting the sparsity and inter-signal correlations among antenna signals. Then, a subspace-projection technique is applied to suppress the signal coefficients related to the wall returns. Furthermore, a multi-task linear model is developed to relate the target coefficients to the image of the scene. The composite image is reconstructed using a joint Bayesian sparse framework, taking into account the inter-view dependencies. Experimental results are presented which demonstrate the effectiveness of the proposed approach for multi-view imaging of indoor scenes using a reduced set of measurements at each view.
\end{abstract}

Keywords: Multi-view through-the-wall radar imaging, wall clutter mitigation, compressed sensing, joint Bayesian sparse recovery.

\section{INTRODUCTION}

Through-the-wall radar imaging (TWRI) is emerging as a powerful technology to capture the content of scenes behind walls and inside enclosed structures. The ability to provide situational awareness behind opaque materials makes TWRI systems highly desirable for law enforcement, search-and-rescue, and emergency operations. ${ }^{1,2}$ In these applications, the behind-the-wall scene is illuminated by transmitting electromagnetic (EM) waves and processing the reflected signals from the wall and targets to form the image. The radar returns, in practice, are typically corrupted by several interferences, such as layover, shadow, and multipath effects which render target detection difficult, if not impossible. For example, when the antenna is placed facing a strong reflective target with another weak target behind, layover effects occur, impeding the detection of the weak target. Furthermore, the reflectivity of the scatterers depends highly on the sensing aspect angle. Target returns may be strong if sensed from the front wall, but may be weak when illuminated from the side wall, and vice versa. The low radar returns result in weak or missing targets in the image formed at a single aspect angle. These problems can be addressed by using multi-view or multi-location sensing and then combining the data acquired from different vantage points to enhance image formation, target detection and localization.

Multi-view TWRI methods typically involve image formation at individual views, followed by image fusion,,$^{2,3}$ target image correction, ${ }^{4}$ or target detection. ${ }^{5}$ These methods, however, require the full data volume at each view to form the images; they are not concerned with the TWRI problem in the compressed sensing (CS) context. ${ }^{6,7}$ CS has emerged as a powerful signal processing technique for efficiently sampling and reconstructing signals. It has been used for TWRI to save data acquisition, reduce computation cost, and improve image formation and fusion. ${ }^{8-11}$ More recently, CS-based techniques have been proposed which combine wall-clutter mitigation with image formation. ${ }^{12-14}$ These methods, however, were proposed for single-view TWRI problem only; they do not consider the inter-view correlations in the imaging model.

In this paper, we address the problem of multi-view TWRI scene reconstruction in the CS context. The aim is to combine multiple compressed measurement sets for enhancing target detection and localization. In the

Email: vht986@uowmail.edu.au, a.bouzerdoum@uow.edu.au,phung@uow.edu.au, tivive@uow.edu.au. 
proposed approach, a joint Bayesian sparse model is first employed to reconstruct the antenna signal coefficients simultaneously, by exploiting both the sparsity and correlations between antenna signals. This joint model differs from the single-signal CS recovery model presented in, ${ }^{12,14}$ where each antenna signal is recovered independently. This paper demonstrates that the proposed joint Bayesian CS model requires far fewer measurements and yields higher reconstruction accuracy than the single-signal CS model. Furthermore, to alleviate wall clutter effects, a subspace projection technique is applied directly to the estimated signal coefficients. Finally, a multitask linear imaging model is developed to relate the clutter-free signal coefficients to the image of the scene. A composite image of the scene is reconstructed using a joint sparse Bayesian learning framework.

The remainder of the paper is structured as follows. Section 2 presents the multi-view TWRI signal model. Section 3 introduces the proposed approach, comprising joint Bayesian antenna signal coefficient estimation, wall-clutter mitigation, and joint Bayesian image reconstruction. Section 4 describes experimental results and analysis. Section 5 gives concluding remarks.

\section{MULTI-VIEW TWRI SIGNAL MODEL}

Consider a monostatic stepped-frequency multi-view TWRI system illuminating a scene behind a wall or inside an enclosed structure. Suppose that the scene containing $P$ targets is imaged at $L$ locations or views, by shifting the same antenna array to new locations vertically or horizontally along the front and side walls. At each view, a transceiver is placed at several scan positions parallel to the wall to synthesize a horizontal $M$-element linear antenna array. The scene is interrogated by transceving a stepped-frequency signal comprising $N$ frequencies, equally spaced over the sensing bandwidth,

$$
f_{n}=f_{0}+n \Delta f, \text { for } n=0,1, \ldots, N-1,
$$

where $f_{n}$ is the $n$-th frequency and $\Delta f$ is the frequency step size. Let $z_{l}(m, n)$ denote the signal of frequency $f_{n}$, received at the $m$-th antenna from the $l$-th view. This signal can be expressed as

$$
z_{l}(m, n)=z_{l}^{w t}(m, n)+\nu_{l}(m, n),
$$

where $z_{l}^{w t}(m, n)$ represents the signal due to the wall and target reflections, and $\nu_{l}(m, n)$ is a noise term. The signal $z_{l}^{w t}(m, n)$ is modeled as the superposition of the wall and target returns,

$$
z_{l}^{w t}(m, n)=z_{l}^{w}(m, n)+z_{l}^{t}(m, n)=\sigma_{w} e^{-j 2 \pi f_{n} \tau_{m, w}}+\sum_{p=1}^{P} \sigma_{p} e^{-j 2 \pi f_{n} \tau_{m, p}},
$$

where $z_{l}^{w}(m, n)$ and $z_{l}^{t}(m, n)$ are the signals representing wall and target returns, respectively, $\sigma_{w}$ is the reflectivity of the wall, $\sigma_{p}$ is the reflectivity of the $p$-th target, $\tau_{m, w}$ is the round-trip travel time of the signal from the $m$-th antenna to the wall, and $\tau_{m, p}$ is the round-trip travel time of the signal from the $m$-th antenna to the $p$-th target.

Assume that the imaged scene is divided into a rectangular grid in crossrange and downrange consisting of $Q$ pixels. Let $s_{l}(q)$ denote a weighted indicator function defined as

$$
s_{l}(q)=s_{l}^{w}(q)+s_{l}^{t}(q)= \begin{cases}\sigma_{w}, & \text { if the wall occupies the } q \text {-th pixel } \\ \sigma_{p}, & \text { if the } p \text {-th target occupies the } q \text {-th pixel } \\ 0, & \text { otherwise }\end{cases}
$$

where $s_{l}^{w}(q)$ and $s_{l}^{t}(q)$ represent for the wall and target components, respectively. We denote by $\mathbf{z}_{l, m}, \mathbf{z}_{l, m}^{w t}$, and $\boldsymbol{\nu}_{l, m}$ the column vectors containing, respectively, the frequency samples $z_{l}(m, n), z_{l}^{w t}(m, n)$, and $\nu_{l}(m, n)$ at the $m$-th antenna for the $l$-th view, see Eq. (2). Similarly, let $\mathbf{s}_{l}, \mathbf{s}_{l}^{w}$, and $\mathbf{s}_{l}^{t}$ be the lexicographically ordered column vector containing the pixel values of the $l$-th view image. It follows from Eqs. (2)-(4) that

$$
\begin{aligned}
& \mathbf{z}_{l, m}=\mathbf{z}_{l, m}^{w t}+\boldsymbol{\nu}_{l, m} \\
& \mathbf{z}_{l, m}^{w t}=\boldsymbol{\Psi}_{l, m} \mathbf{s}_{l}=\boldsymbol{\Psi}_{l, m} \mathbf{s}_{l}^{w}+\Psi_{l, m} \mathbf{s}_{l}^{t}
\end{aligned}
$$


where $\boldsymbol{\Psi}_{l, m}$ is an $N \times Q$ matrix whose $n q$-th element $\Psi_{l, m}(n, q)=e^{-j 2 \pi f_{n} \tau_{m, q}}$, with $\tau_{m, q}$ being the propagation delay between the $m$-th antenna and the $q$-th pixel. By concatenating the received signals at all $M$ antennas, we can write

$$
\begin{aligned}
\mathbf{z}_{l} & =\mathbf{z}_{l}^{w t}+\boldsymbol{\nu}_{l}, \\
\mathbf{z}_{l}^{w t} & =\mathbf{\Psi}_{l} \mathbf{s}_{l},
\end{aligned}
$$

where $\mathbf{z}_{l}=\left[\mathbf{z}_{l, 0}^{T}, \ldots, \mathbf{z}_{l, M-1}^{T}\right]^{T}, \mathbf{z}_{l}^{w t}=\left[\mathbf{z}_{l, 0}^{w t T}, \ldots, \mathbf{z}_{l, M-1}^{w t}\right]^{T}, \mathbf{\Psi}_{l}=\left[\mathbf{\Psi}_{l, 0}^{T}, \ldots, \mathbf{\Psi}_{l, M-1}^{T}\right]^{T}, \boldsymbol{\nu}_{l}=\left[\boldsymbol{\nu}_{l, 0}^{T}, \ldots, \boldsymbol{\nu}_{l, M-1}^{T}\right]^{T}$.

The image of the scene at the $l$-th view $\mathbf{s}_{l}$ can be recovered from (7)-(8) by applying delay-and-sum (DS) beamforming or backprojection. ${ }^{1}$ However, this approach is suitable for single-view TWRI only where the image at each view is reconstructed independently, ignoring the inter-view correlations. Note that before image formation, the wall contributions need to be removed or significantly reduced; otherwise, the wall returns dominate the target reflections, rendering target detection difficult or even impossible. In the next section, we introduce a new approach for compressed multi-view TWRI which incorporates wall clutter mitigation and takes into account the correlations between antenna signals and inter-view dependencies.

\section{JOINT BAYESIAN MULTI-VIEW TWRI MODEL}

This section presents the proposed approach for compressed multi-view TWRI scene reconstruction. First, the antenna signals are represented by a sparsifying dictionary. Then, the signal coefficients are simultaneously estimated using a joint Bayesian sparse framework. Furthermore, a subspace projection technique is applied directly to the estimated coefficients to segregate the wall returns from the target coefficients. Finally, a multitask linear model is developed which combines the single-view measurement vectors to form a composite scene. The single-view scenes and the composite scene are recovered jointly using a Bayesian approach.

\subsection{Joint Signal Coefficient Estimation}

The signal $\mathbf{z}_{l, m}^{w t}$ due to wall and target returns can be sparsely represented using a dictionary $\mathbf{W} \in \mathbb{R}^{N \times R}$ containing $R(R \geq N)$ basis functions or atoms,

$$
\mathbf{z}_{l, m}^{w t}=\mathbf{W} \boldsymbol{\theta}_{l, m}+\boldsymbol{\xi}_{l, m}
$$

where $\boldsymbol{\theta}_{l, m}$ is a vector of signal coefficients and $\boldsymbol{\xi}_{l, m}$ is a vector representing the modeling errors. In compressed multi-view TWRI, the reduced measurements $\mathbf{y}_{l, m}$ collected at the $m$-th antenna can be modeled as

$$
\mathbf{y}_{l, m}=\mathbf{\Phi}_{l, m} \mathbf{z}_{l, m}
$$

where $\boldsymbol{\Phi}_{l, m}$ is a $K \times N$ selection matrix $(K \ll N)$ containing a single unit value in each row and each column. From (5) and (9) it follows that $\mathbf{y}_{l, m}$ can be expressed as

$$
\mathbf{y}_{l, m}=\boldsymbol{\Phi}_{l, m} \mathbf{W} \boldsymbol{\theta}_{l, m}+\boldsymbol{\Phi}_{l, m}\left(\boldsymbol{\xi}_{l, m}+\boldsymbol{\nu}_{l, m}\right)=\mathbf{D}_{l, m} \boldsymbol{\theta}_{l, m}+\boldsymbol{\epsilon}_{l, m},
$$

where $\mathbf{D}_{l, m}=\boldsymbol{\Phi}_{l, m} \mathbf{W}$ and $\boldsymbol{\epsilon}_{l, m}=\boldsymbol{\Phi}_{l, m}\left(\boldsymbol{\xi}_{l, m}+\boldsymbol{\nu}_{l, m}\right)$.

Given the measurement vectors $\mathbf{y}_{l, m}$ and the dictionaries $\mathbf{D}_{l, m}$, the coefficient vectors $\boldsymbol{\theta}_{l, m}$ can be recovered using different approaches. In, ${ }^{9,12}$ the vector $\boldsymbol{\theta}_{l, m}$ is recovered independently at each antenna. These methods, however, do not consider the correlations between antenna signals. In contrast, here we consider the multitask problem (11) as a joint sparsity model, which assumes the coefficient vectors $\boldsymbol{\theta}_{l, m}$ have overlapping support. Here, a sparse Bayesian recovery framework is employed for jointly estimating the coefficient vectors $\boldsymbol{\theta}_{l, m}$ since it is more suitable for the TWRI problem than other simultaneous recovery algorithms. ${ }^{13}$ In the Bayesian model, the noise term in (11) is assumed to be zero-mean Gaussian with independent and identically distributed (i.i.d) components. Thus, the probability density function (pdf) of $\epsilon_{l, m}$ can be expressed as

$$
p\left(\boldsymbol{\epsilon}_{l, m}\right)=\prod_{k=1}^{K} \mathcal{N}\left(\epsilon_{l, m}(k) \mid 0, \beta^{-1}\right),
$$


where $\beta$ is the noise precision (variance $=1 / \beta$ ). The likelihood of $\boldsymbol{\theta}_{l, m}$ is therefore a multivariate Gaussian function,

$$
p\left(\mathbf{y}_{l, m} \mid \boldsymbol{\theta}_{l, m}, \beta\right)=(2 \pi / \beta)^{-K / 2} e^{-\frac{\beta}{2}\left\|\mathbf{y}_{l, m}-\mathbf{D}_{l, m} \boldsymbol{\theta}_{l, m}\right\|^{2}} .
$$

The joint sparsity of the coefficient vectors is enforced using a shared prior imposed on $\boldsymbol{\theta}_{l, m},{ }^{15}$

$$
p\left(\boldsymbol{\theta}_{l, m} \mid \boldsymbol{\alpha}\right)=\prod_{i=1}^{R} \mathcal{N}\left(\theta_{l, m}(i) \mid 0, \alpha_{i}^{-1}\right) .
$$

Given the hyper-parameter vector $\boldsymbol{\alpha}=\left[\alpha_{1}, \ldots, \alpha_{R}\right]$, the posterior of $\boldsymbol{\theta}_{l, m}$ is a multivariate Student-t distribution with mean and covariance given by

$$
\begin{aligned}
\boldsymbol{\mu}_{l, m} & =\Sigma_{l, m} \mathbf{D}_{l, m}^{T} \mathbf{y}_{l, m}, \\
\Sigma_{l, m} & =\left(\mathbf{D}_{l, m}^{T} \mathbf{D}_{l, m}+\mathbf{A}\right)^{-1},
\end{aligned}
$$

where $\mathbf{A}=\operatorname{diag}(\boldsymbol{\alpha})$. The problem now becomes searching for the hyper-parameter vector $\boldsymbol{\alpha}$, which can be obtained by maximizing the logarithm of the marginal likelihood $\mathcal{L}(\boldsymbol{\alpha})$,

$$
\widehat{\boldsymbol{\alpha}}=\arg \max _{\boldsymbol{\alpha}} \mathcal{L}(\boldsymbol{\alpha})=\arg \max _{\boldsymbol{\alpha}} \sum_{m=0}^{M-1} \log p\left(\mathbf{y}_{l, m} \mid \boldsymbol{\alpha}\right) .
$$

The optimization problem in (17) is solved using a fast marginal likelihood maximization method. ${ }^{15-17}$ Once the hyper-parameter vector $\widehat{\boldsymbol{\alpha}}$ is obtained, the coefficient vector $\boldsymbol{\theta}_{l, m}$ is estimated by the mean of the posterior given by

$$
\widehat{\boldsymbol{\theta}}_{l, m}=\left.\boldsymbol{\mu}_{l, m}\right|_{\boldsymbol{\alpha}=\widehat{\boldsymbol{\alpha}}}=\left.\left(\Sigma_{l, m} \mathbf{D}_{l, m}^{T} \mathbf{y}_{l, m}\right)\right|_{\boldsymbol{\alpha}=\widehat{\boldsymbol{\alpha}}}
$$

The reconstructed vector $\widehat{\boldsymbol{\theta}}_{l, m}$ contains coefficients associated with wall returns that usually dominate the target signal. Therefore, before image reconstruction, we need to suppress or remove the coefficients related to the wall returns.

\subsection{Wall Coefficient Mitigation}

Usually, wall-clutter mitigation techniques are applied to the radar signals, ${ }^{18-21}$ which can be estimated from the recovered coefficients $\widehat{\boldsymbol{\theta}}_{l, m}$ as $\widehat{\mathbf{z}}_{l, m}^{w t} \approx \mathbf{W} \widehat{\boldsymbol{\theta}}_{l, m}$, see Eq. (9). Here, however, we apply a subspace projection method directly to the estimated coefficients to segregate the wall contributions from the target returns. Let $\widehat{\boldsymbol{\Theta}}$ denote a matrix having as columns the antenna coefficients $\widehat{\boldsymbol{\theta}}_{l, m}$ obtained from all views. Using singular value decomposition, the matrix $\widehat{\boldsymbol{\Theta}}$ can be expressed as $\widehat{\boldsymbol{\Theta}}=\mathbf{U} \Sigma \mathbf{V}^{H}$, where $\mathbf{U}=\left[\mathbf{u}_{1}, \ldots, \mathbf{u}_{R}\right]$ and $\mathbf{V}=\left[\mathbf{v}_{1}, \ldots, \mathbf{v}_{M L}\right]$ are unitary matrices containing the left and right singular vectors, respectively, and $\Sigma$ is a matrix containing the singular values arranged in descending order along the main diagonal.

In TWRI, the wall returns are relatively stronger than the target reflections. Hence, the wall contributions are captured by the first few singular vectors associated with the dominant singular values. The wall subspace can be defined as

$$
\mathbf{P}_{\mathrm{w}}=\sum_{i \in \mathcal{W}} \mathbf{u}_{i} \mathbf{v}_{i}^{H}
$$

where $\mathcal{W}$ denotes the index set of the singular vectors spanning the wall subspace. ${ }^{20}$ To suppress the wall coefficients, the matrix $\widehat{\boldsymbol{\Theta}}$ is projected onto a subspace orthogonal to the wall subspace:

$$
\widehat{\boldsymbol{\Theta}}^{t}=\left(\mathbf{I}-\mathbf{P}_{\mathrm{w}} \mathbf{P}_{\mathrm{w}}^{H}\right) \widehat{\boldsymbol{\Theta}}
$$

where I denotes the identity matrix. Now the wall-clutter free coefficients $\widehat{\boldsymbol{\Theta}}^{t}$, which contains in its columns the target coefficient vectors $\widehat{\boldsymbol{\theta}}_{l, m}^{t}$, can be used for image reconstruction. 


\subsection{Joint Bayesian Sparse Scene Reconstruction}

The scene can be formed by first reconstructing the radar signal from the target coefficients $\widehat{\boldsymbol{\theta}}_{l, m}^{t}$, see Eq. (9), and then applying DS beamforming ${ }^{9}$ or $\ell_{1}$ minimization. ${ }^{12,14}$ However, these methods are designed for singleview image formation, which ignores the inter-view dependencies. Here, we formulate a multitask linear model that maps the clutter-free coefficients to the corresponding images of the scene and incorporates a composite coefficient vector representing the fused image of the scene. Using (6) and (9), we can relate the target coefficients to the target image as follows:

$$
\widehat{\boldsymbol{\theta}}_{l, m}^{t}=\mathbf{W}^{-1}\left(\boldsymbol{\Psi}_{l, m} \mathbf{s}_{l}^{t}-\boldsymbol{\xi}_{l, m}\right)=\widehat{\mathbf{\Psi}}_{l, m} \mathbf{s}_{l}^{t}+\widehat{\boldsymbol{\xi}}_{l, m},
$$

where $\widehat{\boldsymbol{\Psi}}_{l, m}=\mathbf{W}^{-1} \mathbf{\Psi}_{l, m}$, and $\widehat{\boldsymbol{\xi}}_{l, m}=-\mathbf{W}^{-1} \boldsymbol{\xi}_{l, m}$. By stacking the coefficients belonging to the $l$-th view, we can rewrite

$$
\widehat{\boldsymbol{\theta}}_{l}^{t}=\widehat{\mathbf{\Psi}}_{l} \mathbf{s}_{l}^{t}+\widehat{\boldsymbol{\xi}}_{l}
$$

where $\widehat{\boldsymbol{\theta}}_{l}^{t}=\left[\widehat{\boldsymbol{\theta}}_{l, 0}^{t}{ }^{T}, \ldots, \widehat{\boldsymbol{\theta}}_{l, M-1}^{t}\right]^{T}, \widehat{\mathbf{\Psi}}_{l}=\left[\widehat{\mathbf{\Psi}}_{l, 0}^{T}, \ldots, \widehat{\mathbf{\Psi}}_{l, M-1}^{T}\right]^{T}$, and $\widehat{\boldsymbol{\xi}}_{l}=\left[\widehat{\boldsymbol{\xi}}_{l, 0}^{T}, \ldots, \widehat{\boldsymbol{\xi}}_{l, M-1}^{T}\right]^{T}$.

Because the vectors $\mathbf{s}_{l}^{t}$ represent images of the same target scene, a final composite image can be obtained using image fusion techniques after each single-view image has been reconstructed and aligned. ${ }^{2,22}$ Here instead, we propose to first combine the coefficient vectors from different views, then perform fusion using joint Bayesian sparse learning. Since the imaging coordinates are different between views, we need to adopt a pixel scanning scheme in which all the vectors $\mathbf{s}_{l}^{t}$ have the same sparsity support. ${ }^{23}$ Therefore, a linear imaging model relating a composite coefficient vector $\boldsymbol{\theta}^{t}$ to the fused image of the scene $\mathbf{s}^{t}$ can be formulated as a linear combination of the coefficient vectors of different views:

$$
\boldsymbol{\theta}^{t}=\sum_{l=1}^{L} w_{l} \widehat{\boldsymbol{\theta}}_{l}^{t}=\boldsymbol{\Psi} \mathbf{s}^{t}+\boldsymbol{\xi},
$$

where $w_{l}$ 's are positive weights $\left(\sum_{l=1}^{L} w_{l}=1\right)$ computed based on mutual information $(\mathrm{MI}),{ }^{11} \mathbf{\Psi}=\sum_{l=1}^{L} w_{l} \widehat{\boldsymbol{\Psi}}_{l}$, and $\boldsymbol{\xi}=\sum_{l=1}^{L} w_{l} \widehat{\boldsymbol{\xi}}_{l}$. By combining this composite linear model with (22), we obtain an overall multitask model for the multi-view TWRI problem:

$$
\widehat{\boldsymbol{\theta}}_{l}^{t}=\widehat{\mathbf{\Psi}}_{l} \mathbf{s}_{l}^{t}+\widehat{\boldsymbol{\xi}}_{l}, l=1, \ldots,(L+1)
$$

where $\widehat{\boldsymbol{\theta}}_{L+1}^{t}=\boldsymbol{\theta}^{t}, \widehat{\mathbf{\Psi}}_{L+1}=\boldsymbol{\Psi}, \mathbf{s}_{L+1}^{t}=\mathbf{s}^{t}$, and $\widehat{\boldsymbol{\xi}}_{L+1}=\boldsymbol{\xi}$. The solution of (24) yields $L+1$ images corresponding to the $L$ individual views plus a composite image of the scene. This multitask problem can be solved efficiently using the joint Bayesian sparse model, which exploits inter-view correlations.

\section{EXPERIMENTAL RESULTS AND ANALYSIS}

In this section, we present the experimental results obtained using numerical EM simulation data. Experimental analysis and comparison with existing compressed TWRI models are also provided.

\subsection{Experimental Setup}

The simulation is based on XFDTD, a full-wave EM simulator. We build a computer model of an enclosed structure scene containing three dihedral targets. Dihedral 1 and Dihedral 2 have orientation angle facing the front wall, whereas Dihedral 3 has its corner oriented towards the side wall. The scene behind a concrete wall is illuminated from two different aspect angles: $0^{\circ}$ view (through the front wall) and $90^{\circ}$ view (through the side wall of the enclosed structure), see Fig. 1. At each view, the transceiver is placed at 51 positions parallel to the wall at a standoff distance of $1 \mathrm{~m}$, to synthesize an array aperture of length $1.2 \mathrm{~m}$. The transmitted frequency range is $1 \mathrm{GHz}$, centered at $2.5 \mathrm{GHz}$, with a step frequency of $3 \mathrm{MHz}$ (i.e. 334 frequency bins). For sparsifying the signals, the dictionary $\mathbf{W}$ is constructed using modulated discrete prolate spheroidal sequences (DPSS) since the DPSS dictionary is overcomplete and can represent bandpass radar signals more compactly in the range profile than does the Fourier basis. ${ }^{14}$ 

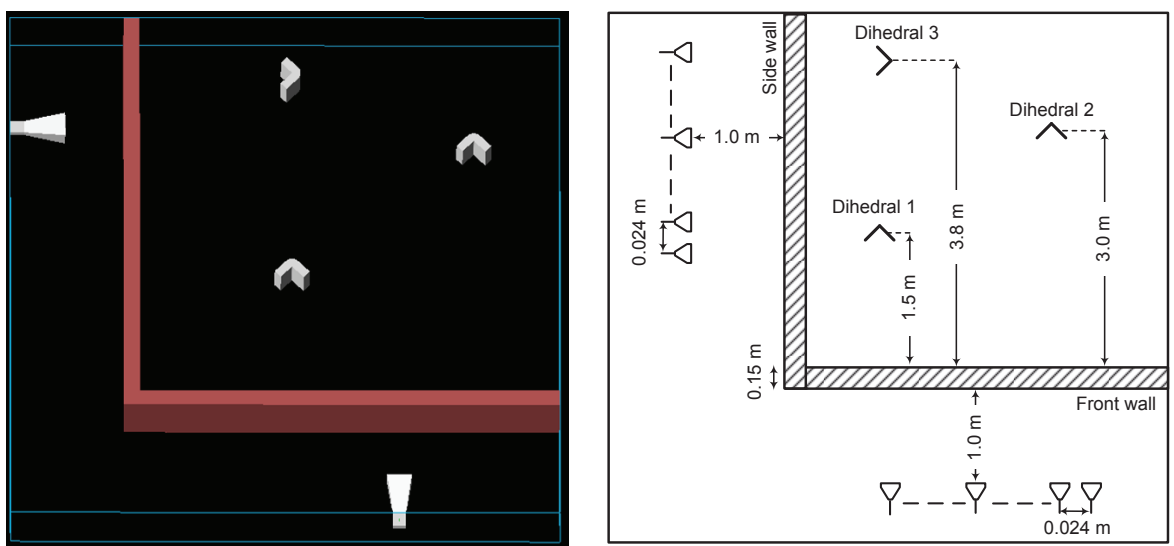

Figure 1. Multi-view TWRI data acquisition scene. Left: a photo of the scene; Right: a top-view of the behind-the-wall scene.

The normalized mean squared error is used to measure the accuracy of the signal recovery:

$$
\mathrm{NMSE}=\|\mathbf{z}-\hat{\mathbf{z}}\|_{2} /\|\mathbf{z}\|_{2},
$$

where $\hat{\mathbf{z}}$ and $\mathbf{z}$ are the reconstructed and true signals, respectively. The image quality is measured using the target-to-clutter ratio (in $\mathrm{dB}$ ):

$$
\mathrm{TCR}=10 \log _{10}\left(P_{\text {target }} / P_{\text {clutter }}\right),
$$

where $P_{\text {target }}$ and $P_{\text {clutter }}$ are the average power in the target and clutter regions, respectively. The receiver operating characteristic (ROC) curve is used to measure the probability of target detection for a given false alarm rate. The probability of detection, or detection rate, denotes the percentage of pixels in target regions that are correctly detected. By contrast, the probability of false alarm, or false alarm rate, is the percentage of pixels in the clutter region that are incorrectly detected as targets.

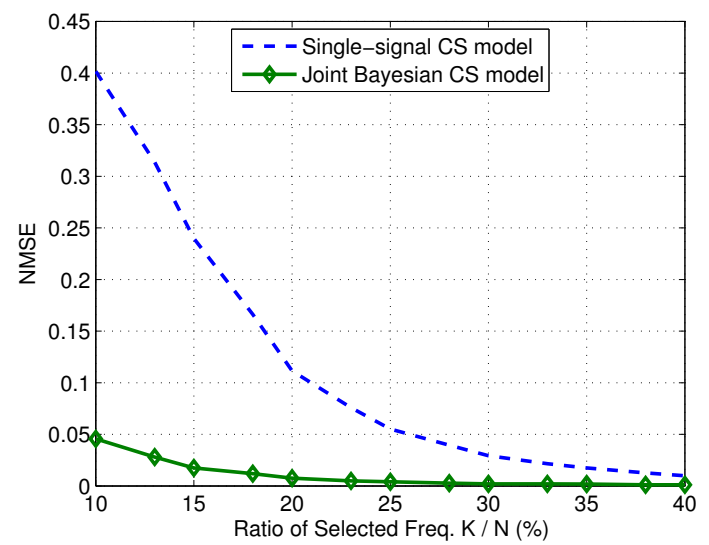

Figure 2. The NMSE for signal reconstruction using the single-signal CS model and the joint Bayesian CS model.

\subsection{Results and Analysis}

In the first experiment, we used half of the antennas and varied the selected frequencies from $10 \%$ to $40 \%$ of the full frequencies at each view. For each set of measurements, the signals were recovered by the single-signal CS model and joint Bayesian CS model. The NMSE was recorded for 100 trials. Figure 2 shows the average NMSE for both models. Compared to the single-signal CS model, the proposed joint Bayesian CS model produces a considerably lower reconstruction error, especially when the number of measurements is very low. Moreover, to 


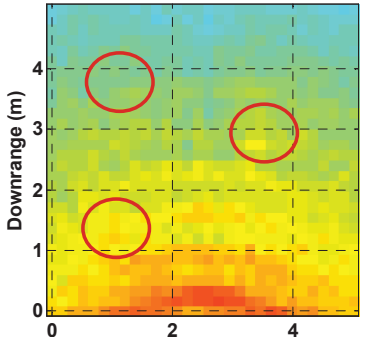

(a) -Front wall

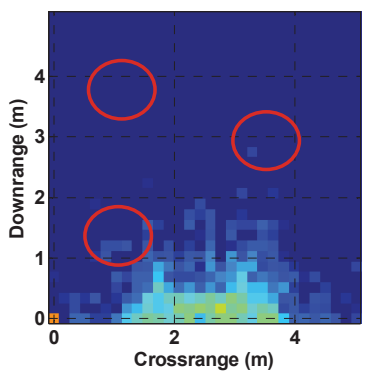

(d) -Front wall

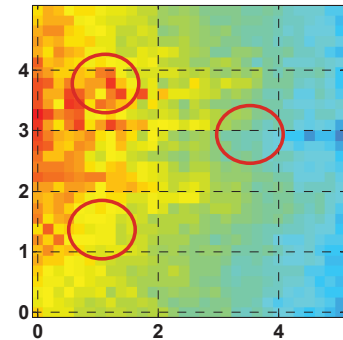

(b) —Side wall

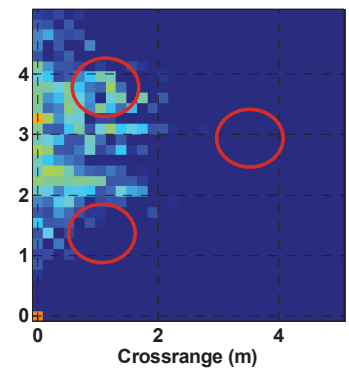

(e) - Side wall

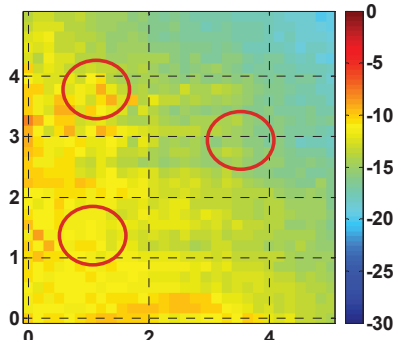

(c)-Composite image

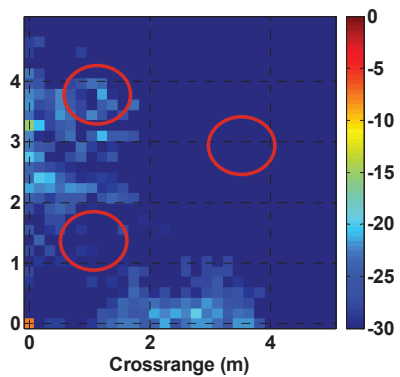

(f)-Composite image

Figure 3. Images reconstructed from signals recovered by single-signal CS model: (a)-(c) images reconstructed using DS beamforming, (d)-(f) images reconstructed separately using standard CS model.

obtain the same reconstruction accuracy, the proposed approach requires far fewer measurements than does the single-signal CS model. For example, to obtain an NMSE $=0.05$, the joint Bayesian sparse approach requires only $10 \%$ of the frequency measurements, whereas the single-signal CS model uses $25 \%$. The superiority of the reconstruction by the proposed joint Bayesian model is due to the fact that it exploits the signal sparsity and the correlations among the signals, whereas the conventional single-signal CS model considers only the signal sparsity.

In the second experiment, the signals recovered using $20 \%$ of the total measurements are used for scene reconstruction, after wall clutter mitigation. Figure 3 shows the images formed by different methods using the signals recovered with the single-signal CS model. Figures 3(a)-(b) present the front-view and side-view images formed using DS beamforming, averaged over 100 trials; these images contain heavy clutter and very weak targets. Figure 3(c) presents the composite image obtained from the images of Figs. 3(a) and (b) using MI-based fusion. Clearly, the targets are not detectable, even in the fused image. Figures $3(\mathrm{~d})-(\mathrm{f})$ illustrate the images obtained separately from the front view, side view, and their fused image, respectively, using the conventional CS reconstruction. Again, it is impossible to localize all the targets.

Figure 4 shows the scene images formed by different imaging methods using the signals jointly recovered by the proposed Bayesian sparse model. Figures 4(a)-(c) present, respectively, the front-view, side-view, and composite images reconstructed using standard CS model. Note that the composite image is formed by combining the measurements from the two views, but these images are recovered separately using the existing single-view CS method. The single-view images in Figs. 4(a) and (b) do not provide a complete picture of the scene content: Dihedrals 1 and 2 are weak in the side view, and Dihedral 3 is week in the frontal view. Moreover, we can observe the appearance of outliers in the composite image shown in Fig. 4(c). By contrast, Figs. 4(d)-(f) show the images formed by the joint Bayesian sparse model; they contain much less clutter and reveal all the targets. The TCRs of the composite scene images formed by the conventional CS and the proposed Bayesian approach are, respectively, $15.02 \mathrm{~dB}$ and $34.68 \mathrm{~dB}$. Figure 5 illustrates the ROC curves of the different imaging models. This figure shows that by reconstructing multiple images jointly and exploiting the inter-view dependencies, target detection is significantly enhanced, compared with the methods that form the images individually at each view. 


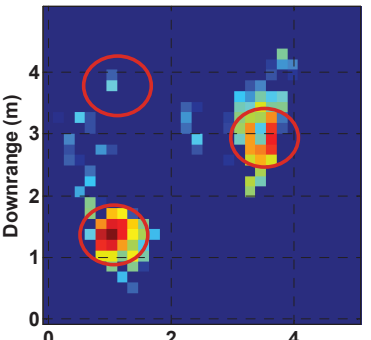

(a) $\stackrel{2}{\text { FFront wall }}$

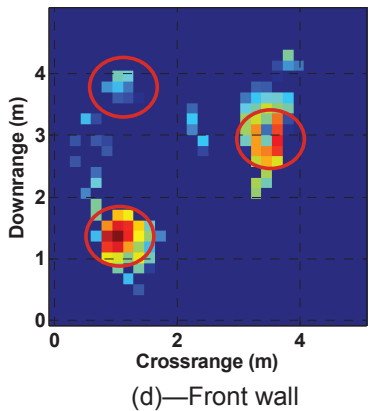

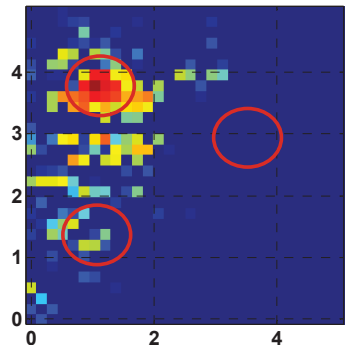

(b) ${ }^{2}$ Side wall

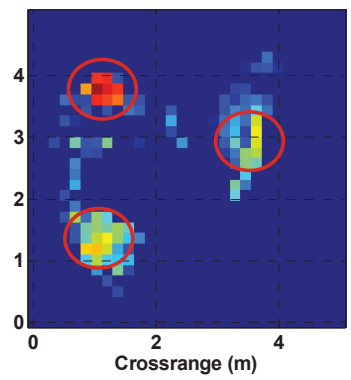

(e)-Side wall

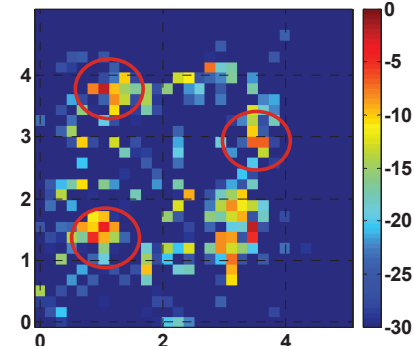

(c)-Composite image

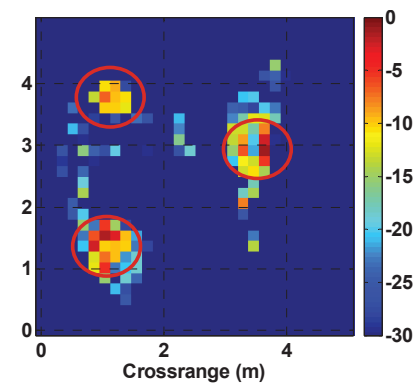

(f)-Composite image

Figure 4. The scene reconstructed using signals jointly recovered by the proposed Bayesian model: (a)-(c) images reconstructed separately using standard CS model, (d)-(f) images formed using joint Bayesian sparse reconstruction.

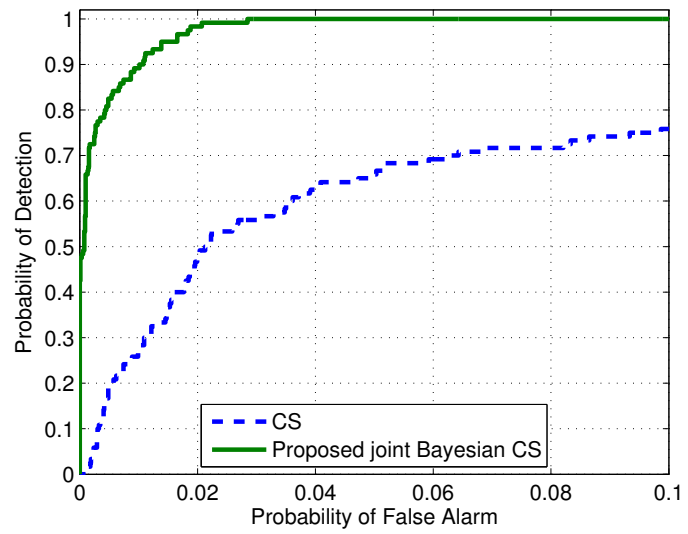

Figure 5. ROC curves of composite images formed by standard CS model and proposed joint Bayesian CS model.

\section{CONCLUSION}

This paper presented a new approach for compressed multi-view TWRI using a joint Bayesian sparse approximation model. In the proposed approach, a joint Bayesian sparse framework is employed for estimating simultaneously the signal coefficients of different antennas from a reduced set of measurements. This joint Bayesian estimation exploits the sparsity and correlation among antenna signals, thereby improving signal reconstruction accuracy. A subspace projection technique is applied directly to the recovered coefficients to segregate wall reflections from the target returns. Furthermore, a multitask imaging model is developed which relates the target coefficients to the images of the scene. Individual images from each view and a composite image of the scene are reconstructed jointly using a sparse Bayesian approximation model, taking into account the inter-view dependencies. Experimental results show that the proposed approach enhances image reconstruction, target detection and localization, compared with forming images separately for each view. 


\section{Acknowledgments}

This work was supported in part by a grant from the Australian Research Council.

\section{REFERENCES}

[1] Amin (Ed.), M. G., [Through-The-Wall Radar Imaging], Boca Raton, FL: CRC Press (2010).

[2] Seng, C. H., Bouzerdoum, A., Amin, M. G., and Phung, S. L., "Probabilistic fuzzy image fusion approach for radar through wall sensing," IEEE Trans. Image Processing 22(12), 4938-4951 (Dec. 2013).

[3] Ahmad, F. and Amin, M. G., "Multi-location wideband synthetic aperture imaging for urban sensing applications," Journal of the Franklin Institute 345(6), 618-639 (Sep. 2008).

[4] Wang, G. and Amin, M. G., "Imaging through unknown walls using different standoff distances," IEEE Trans. Signal Processing 54(10), 4015-4025 (Oct. 2006).

[5] Debes, C., Amin, M. G., and Zoubir, A. M., "Target detection in single- and multiple-view through-the-wall radar imaging," IEEE Trans. Geoscience and Remote Sensing 47(5), 1349-1361 (May 2009).

[6] Donoho, D. L., "Compressed sensing," IEEE Trans. Information Theory 52(4), 1289-1306 (Apr. 2006).

[7] Candes, E. J., Romberg, J., and Tao, T., "Stable signal recovery from incomplete and inaccurate measurements," Communications on Pure and Applied Mathematics 59(8), 1207-1223 (Aug. 2006).

[8] Huang, Q., Qu, L., Wu, B., and Fang, G., "UWB through-wall imaging based on compressive sensing," IEEE Trans. Geoscience and Remote Sensing 48(3), 1408-1415 (Mar. 2010).

[9] Yoon, Y.-S. and Amin, M. G., "Through-the-wall radar imaging using compressive sensing along temporal frequency domain," in [Proc. IEEE Int. Conf. Acoustics, Speech, and Signal Processing], 2806-2809, Dallas, TX (Mar. 2010).

[10] Tang, V. H., Bouzerdoum, A., and Phung, S. L., "Two-stage through-the-wall radar image formation using compressive sensing," Journal of Electronic Imaging 22(2), 021006-1-021006-10 (Apr.--Jun. 2013).

[11] Bouzerdoum, A., Yang, J., and Tivive, F. H. C., "Compressive sensing for multi-polarization through-thewall radar imaging," in [Compressive Sensing for Urban Radar], 232-248, M. G. Amin (Ed.), Boca Raton, FL: CRC Press (Aug. 2014).

[12] Lagunas, E., Amin, M. G., Ahmad, F., and Najar, M., "Joint wall mitigation and compressive sensing for indoor image reconstruction," IEEE Trans. Geoscience and Remote Sensing 51(2), 891 - 906 (Feb. 2013).

[13] Tang, V. H., Bouzerdoum, A., Phung, S. L., and Tivive, F. H. C., "Enhanced wall clutter mitigation for through-the-wall radar imaging using joint Bayesian sparse signal recovery," in [Proc. IEEE Int. Conf. Acoustics, Speech and Signal Processing], 7804-7808, Florence, Italy (May 2014).

[14] Ahmad, F., Qian, J., and Amin, M. G., "Wall clutter mitigation using discrete prolate spheroidal sequences for sparse reconstruction of indoor stationary scenes," IEEE Trans. Geoscience and Remote Sensing 53(3), 1549-1557 (Mar. 2015).

[15] Ji, S., Dunson, D., and Carin, L., "Multitask compressive sensing," IEEE Trans. Signal Processing 57(1), 92-106 (Jan. 2009).

[16] Tipping, M. E. and Faul, A. C., "Fast marginal likelihood maximisation for sparse Bayesian models," in [Proc. the Ninth Inter. Workshop on Artificial Intelligence and Statistics], 3-6, Key West, FL (Jan. 2003).

[17] Wu, Q., Zhang, Y. D., Amin, M. G., and Himed, B., "Complex multitask Bayesian compressive sensing," in [Proc. IEEE Int. Conf. Acoustics, Speech and Signal Processing], 3375-3379, Florence, Italy (May 2014).

[18] Yoon, Y.-S. and Amin, M. G., "Spatial filtering for wall-clutter mitigation in through-the-wall radar imaging," IEEE Trans. Geoscience and Remote Sensing 47(9), 3192-3208 (Sep. 2009).

[19] Tivive, F. H. C., Bouzerdoum, A., and Amin, M. G., "An SVD-based approach for mitigating wall reflections in through-the-wall radar imaging," in [Proc. IEEE Radar Conf.], 519-524, Kansas City, MO (May 2011).

[20] Tivive, F. H. C. and Bouzerdoum, A., "An improved SVD-based wall clutter mitigation method for throughthe-wall radar imaging," in [Proc. IEEE Workshop on Signal Processing Advances in Wireless Communications], 430-434, Darmstadt, Germany (June 2013).

[21] Tivive, F. H. C., Bouzerdoum, A., and Amin, M. G., "A subspace projection approach for wall clutter mitigation in through-the-wall radar imaging," IEEE Trans. Geoscience and Remote Sensing 53(4), 21082122 (Apr. 2015). 
[22] Seng, C. H., Bouzerdoum, A., Amin, M. G., and Phung, S. L., "Two-stage fuzzy fusion with applications to through-the-wall radar imaging," IEEE Geoscience and Remote Sensing Letters 10(4), 687-691 (July 2012).

[23] Yang, J., Bouzerdoum, A., and Amin, M. G., "Multi-view through-the-wall radar imaging using compressed sensing," in [Proc. European Signal Processing Conference], 1429-1433, Aalborg, Denmark (Aug. 2010). 